Research Paper

\title{
A Phase II Study of Concurrent Docetaxel, Epirubicin and Cyclophospha- mide as a Neoadjuvant Chemotherapy Regimen in Patients with Locally Advanced Breast Cancer
}

\author{
Xin Yao, Janet Hosenpud, Christopher R. Chitambar, John Charlson, Yee Chung Cheng ${ }^{凶}$ \\ Division of Hematology and Oncology, Department of Medicine, Medical College of Wisconsin, Milwaukee, Wisconsin, USA
}

Corresponding author: Yee Chung Cheng, M.D., Division of Hematology and Oncology, Department of Medicine, Medical College of Wisconsin, 9200 W Wisconsin Ave, Milwaukee, Wisconsin 53226. Tel: 414-805-4600; Fax: 414-805-4606; Email: ycheng@mcw.edu

(c) Ivyspring International Publisher. This is an open-access article distributed under the terms of the Creative Commons License (http:/ / creativecommons.org/ licenses/by-nc-nd/3.0/). Reproduction is permitted for personal, noncommercial use, provided that the article is in whole, unmodified, and properly cited.

Received: 2011.12.18; Accepted: 2012.02.11; Published: 2012.04.01

\begin{abstract}
Background: Neoadjuvant chemotherapy with concurrent docetaxel, doxorubicin and cyclophosphamide is commonly used for patients with locally advanced breast cancer. Epirubicin is another anthracycline used in breast cancer but the concurrent use of epirubicin and taxane is not well-established. We conducted a single institution, phase II study to assess the efficacy and safety of concurrent docetaxel, epirubicin and cyclophosphamide (TEC) as a neoadjuvant chemotherapy regimen in breast cancer. Methods: Patients with newly diagnosed locally advanced breast cancer defined as $\mathrm{T} 2>3 \mathrm{~cm}, \mathrm{~T} 3, \mathrm{~T} 4$ with any $\mathrm{N}$, or any $\mathrm{T}$ with $\mathrm{NI}-3$ were eligible. A chemotherapy regimen of docetaxel $75 \mathrm{mg} / \mathrm{m}^{2}$, epirubicin $75 \mathrm{mg} / \mathrm{m}^{2}$ and cyclophosphamide $600 \mathrm{mg} / \mathrm{m}^{2}$ was given with filgrastim support every 3 weeks for 6 cycles. The primary end-point was pathologic complete response rate. Results: Twenty patients were enrolled from 2003 to 2006. The median age was 5 I (29-70) year-old. Eight patients were premenopausal. Ten patients had positive hormone receptors. Four patients had HER2 positive receptor. Nineteen patients completed six cycles of TEC chemotherapy. The pathologic complete response rate was $25 \%$. Eight of sixteen patients with NI-3 disease had pathological negative lymph nodes. With a median follow up of 57.5 (I6-7I) months, four patients relapsed including one death from recurrence. The estimated 5 year relapse-free survival was $79.3 \%$ and the 5 -year overall survival was $94.7 \%$. No patient had cardiac failure or death during treatment. The most common grade 3-4 toxicity was neutropenia (35\%). Conclusion: TEC regimen is a well- tolerated and effective neoadjuvant chemotherapy regimen for locally advanced breast cancer that results in a pathologic complete response rate of $25 \%$.
\end{abstract}

Key words: Neoadjuvant Chemotherapy, Docetaxel, Epirubicin, Cyclophosphamide, Locally Advanced, Breast Cancer

\section{Introduction}

Neoadjuvant chemotherapy is a standard of care for patients with locally advanced breast cancer. Compared to adjuvant therapy in which there is no measurable disease, the primary tumor can be used as a surrogate marker of occult metastatic disease re- sponse in the setting of neoadjuvant therapy. The hypothesis is that if the primary tumor responds well to systemic chemotherapy, the systemic micrometastatic disease should respond as well and will result in improved survival. The change in tumor size can be 
assessed by either a clinical examination or by imaging studies. Therefore, neoadjuvant chemotherapy allows the clinician to perform a real-time assessment of tumor response and offers the flexibility of changing therapy if patients do not show an adequate response to the current chemotherapy. ${ }^{1}$ Several studies have demonstrated that the pathological response to neoadjuvant therapy is a marker for long-term survival. $^{2}$ In addition, neoadjuvant therapy provides a platform to identify new molecular and genetic markers to predict tumor response.

There is no standard neoadjuvant chemotherapy regimen. In the United States, four cycles of doxorubicin and cyclophosphamide (AC) followed by four cycles of taxane (paclitaxel or docetaxel) is commonly used. In Canada and Europe, fluorouracil, epirubicin and cyclophosphamide for 6 cycles is more popular. The addition of taxane augments the efficacy of anthracycline-based chemotherapy regimen. The NSABP-B27 study demonstrated that preoperative administration of docetaxel following AC increased the pathological complete response $(\mathrm{pCR})$ rate from $13.7 \%$ to $26.1 \% .^{2}$ An M.D. Anderson study showed adding paclitaxel prior to preoperative fluorouracil, doxorubicin and cyclophosphamide regimen increased a pCR rate from $15.7 \%$ to $28.2 \% .^{3}$ Compared to sequential treatment, concurrent use of taxane with anthracycline may provide a similar response but with shorten treatment duration. The GeparTrio trial found that the six cycles of docetaxel, doxorubicin and cyclophosphamide (TAC) produced a pCR of $21 \%$ if the primary tumor size decreased by $\geq 50 \%$ and a $\mathrm{pCR}$ of $5.3 \%$ if the decrease was $<50 \%$ after two cycles of chemotherapy. ${ }^{1,4}$ Epirubicin has comparable efficacy to doxorubicin but less cardiotoxicity. Theoretically, the concurrent administration of docetaxel, epirubicin and cyclophosphamide (TEC) should be as effective as TAC regimen but may have a less toxicity profile. The use of TEC regimen as neoadjuvant chemotherapy has not been well-established. We conducted a single institution phase II study to assess the efficacy and safety of neoadjuvant TEC regimen in patients with locally advanced breast cancer.

\section{Patients and Methods}

Patient population: All patients signed an informed consent form. The inclusion criteria were female patients with histologically confirmed breast cancer; either T2 $>3 \mathrm{~cm}, \mathrm{~T} 3, \mathrm{~T} 4$ with any $\mathrm{N}$, or any $\mathrm{T}$ with N1-3, and M0. Patients were registered within 45 days of diagnosis. The age was $\geq 18$ years old. Patients were required to have Eastern Cooperative Oncology Group performance status scale $0-1$ and were candidate for systemic chemotherapy. The exclusion crite- ria were patients who were pregnant or lactating, had undergone lumpectomy, had received any prior systemic or radiation therapy for the breast cancer, had any other serious medical or psychiatric illness, had any history of chronic liver disease, active infection, prior malignancies except non-melanoma skin cancer, in-situ carcinoma of the cervix, or other non-breast cancer if disease free $>5$ years.

Biopsy: Patients with palpable axillary lymph nodes underwent an excisional or core needle biopsy of the nodes. If there was no palpable disease, the patient underwent standard pre-surgical radio-lymphatic mapping followed by sentinel lymph node biopsy.

Initial evaluation: All patients had whole body bone scan, computed tomography scan of chest, abdomen and pelvis to rule out distant metastasis. Patients had ultrasound, mammography, or magnetic resonance imaging to determine the longest diameter of the primary breast tumor. Patients had a left ventricular ejection fraction measurement by either MUGA scan or Echocardiogram. For patients who were of childbearing potential, negative pregnancy test was documented.

Neoadjuvant chemotherapy: All eligible patients received 6 cycles of neoadjuvant chemotherapy consisting of docetaxel $75 \mathrm{mg} / \mathrm{m}^{2}$, epirubicin $75 \mathrm{mg} / \mathrm{m}^{2}$ and cyclophosphamide $600 \mathrm{mg} / \mathrm{m}^{2}$ every 3 weeks. All three agents were given on day 1 of each cycle. Patients were offered either filgrastim $5 \mu \mathrm{g} / \mathrm{kg}$ subcutaneously on day 5-15 or pegfilgrastim $6 \mu \mathrm{g}$ subcutaneously on day 2 . To receive chemotherapy, patients must have absolute granulocyte count $>1,500 / \mu \mathrm{l}$, platelet count $>100,000 / \mu \mathrm{l}$, hemoglobin $>10 \mathrm{~g} / \mathrm{dl}$, creatinine $<1.5 \mathrm{x}$ upper limit normal (ULN), AST $<1.5$ $x$ UNL, alkaline phosphates $<5 \times$ UNL, and total bilirubin < ULN. Administration of chemotherapy might be delayed because of hematological, renal or gastrointestinal toxicity on day 1 of any cycle. No dose reduction was allowed for hematological toxicities only.

Tumor size was assessed after two cycles of chemotherapy. If the tumor remained stable or shrank, the patient would proceed with another four cycles of chemotherapy treatment. If the patient had progressive disease, she would then be removed from the study and further treatment would be given according to the best standard of care.

Surgery, radiation and other therapy: Three to six weeks after completion of last cycle of neoadjuvant chemotherapy, the patients underwent primary surgical resection. Surgery for the primary tumor was either lumpectomy or mastectomy and the choice was left to the surgeon according to best practice standards or available protocols. Axillary lymph node dis- 
section was performed on all patients with nodal involvements. All lumpectomy patients had adjuvant radiation therapy. The radiation oncologist determined the need for chest wall radiation for mastectomy patients and / or axillary radiation according to best practice standards. Adjuvant anti-hormone therapy was given to patients with positive hormone receptors. Adjuvant trastuzumab was given if the tumor was HER2 receptor positive. After surgery, patients were followed every 3 months for 2 years and then every 6 months for another 3 years. Further disease follow up was at the discretion of the treating physician.

Toxicity: All patients who received neoadjuvant chemotherapy were evaluated for treatment related toxicity. Adverse events were summarized using the Common Toxicity Criteria of the National Cancer Institute for Adverse Events, version 3.0.

Statistical analysis: The primary endpoint of the study was $\mathrm{pCR}$ rate. The $\mathrm{pCR}$ was defined as no histological evidence of invasive tumor cells in the surgical breast specimen and axillary lymph nodes. The secondary endpoints were clinical response to neoadjuvant chemotherapy, adverse events, relapse-free survival (RFS) and overall survival (OS). The primary tumors were evaluated by imaging and physical examination at baseline, after the second cycle and the last cycle of chemotherapy. RECIST guidelines were used to document clinical response. RFS was defined as the time from the date of histological diagnosis to the first date of disease relapse or the date of death of any cause. OS was defined as the time from the date of diagnosis to the date of death of any cause.

A $25 \%$ of pCR rate and a $70 \%$ of clinical response rate was anticipated. Approximately 40 patients were planned to be enrolled into this protocol to produce a confidence interval of $53.5 \%$ to $83.4 \%$. The study was terminated early after an interim analysis was conducted half way through the proposal. The RFS and OS were estimated using the Kaplan-Meier analysis.

\section{Results}

Patient characteristics: Twenty one female patients were registered from Dec 2003 to Dec 2006. One patient was excluded because of a history of papillary thyroid cancer. Twenty patients were evaluated (Table 1). The median age was 51 year-old (range 29-70). Eight of them $(40 \%)$ were premenopausal. Two patients had invasive lobular carcinoma and the others had invasive ductal carcinoma. One patient had inflammatory breast cancer. The median size of the primary tumor was 5.5 (range $0-11$ ) $\mathrm{cm}$ by physical examination and 3.3 (range 1.5-12) $\mathrm{cm}$ by imaging studies. Sixteen patients $(80 \%)$ had axillary lymph node involvements. Ten patients $(50 \%)$ had positive hormone receptors. Four patients $(20 \%)$ were HER2 receptor positive. All but one patient received adjuvant radiation therapy. All patients with positive hormone receptor received adjuvant hormonal treatment at a median duration of 52 (range 12-60) months. The 4 patients with HER2 positive tumor also received 12 months adjuvant trastuzumab treatment.

Table I. Baseline clinical characteristics of the study population $(\mathrm{N}=20)$

\begin{tabular}{ll}
\hline Characteristic & No. \\
\hline Age, years & 51 \\
Mean & $29-70$ \\
range & \\
Menopausal status & 8 \\
premenopausal & 12 \\
postmenopausal & \\
Clinical stage & 12 \\
T2 & 7 \\
T3 & 1 \\
T4 & \\
Clinical nodal status & 4 \\
N0 & 13 \\
N1 & 3 \\
N2 & \\
Scarf-Bloom-Richardson tumor grade & 3 \\
I & 9 \\
II & 8 \\
III & \\
Tumor hormone receptor status & 10 \\
Positive & 10 \\
negative & \\
Tumor HER2 receptor status & 4 \\
positive & \\
negative & \\
\hline & \\
\hline
\end{tabular}

Outcome: Fourteen patients $(70 \%)$ had lumpectomy and six patients $(30 \%)$ underwent mastectomy. For three of these six patients, mastectomy was done because of presence of residual disease. Two mastectomies were performed because patients had multicentric disease. One patient was offered lumpectomy but she decided to have mastectomy.

Nineteen patients (95\%) showed primary tumor shrinkage measured by either clinical examination or imaging studies after six cycles of TEC chemotherapy. Five patients had pCR (25\%). Sixteen patients with nodal involvement had lymph node dissection after 
completion of chemotherapy; eight of them (50\%) had pathological negative lymph nodes.

With a median 57.5 (range 16-71) months follow-up, four patients $(20 \%)$ relapsed. All of them had lymph nodes involvement at diagnosis and failed to achieve $\mathrm{pCR}$. Two of them had triple negative disease, one patient was ER-PR-HER2 ${ }^{+}$and another one was ER $^{+} \mathrm{PR}^{+}{ }^{+}$HER2- . The median time of relapse was 43 months (range 22-50). One patient had a relapse at 22 months and died at 24 months after diagnosis. One relapsed patient transferred her care to another institution. One patient who was in remission at 22 months moved out and lost follow up. All other patients remained alive at the latest follow up. The estimated 5-year RFS was $79.3 \%$ (Figure 1) and the 5 -year OS was $94.7 \%$ (Figure 2).

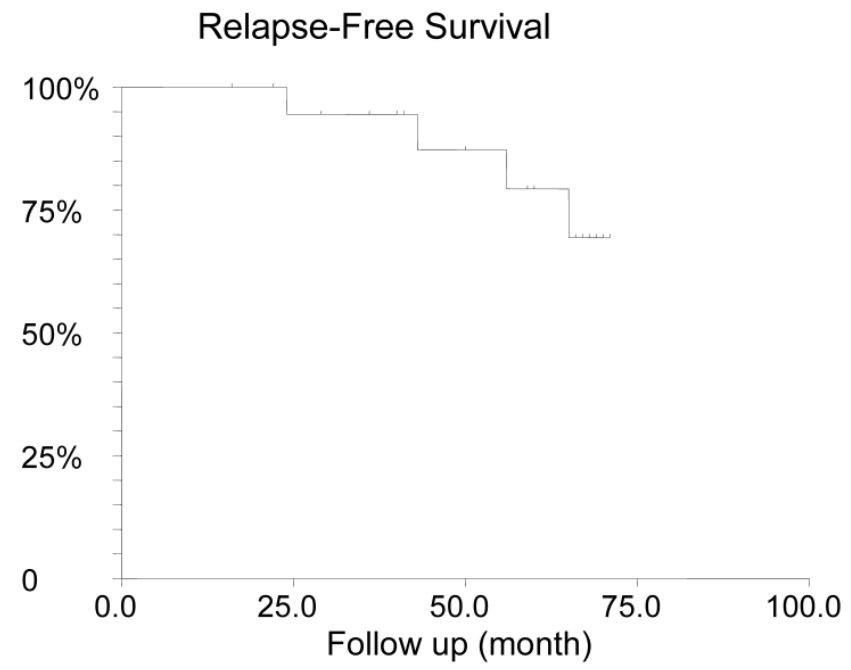

Figure I. Relapse-Free Survival

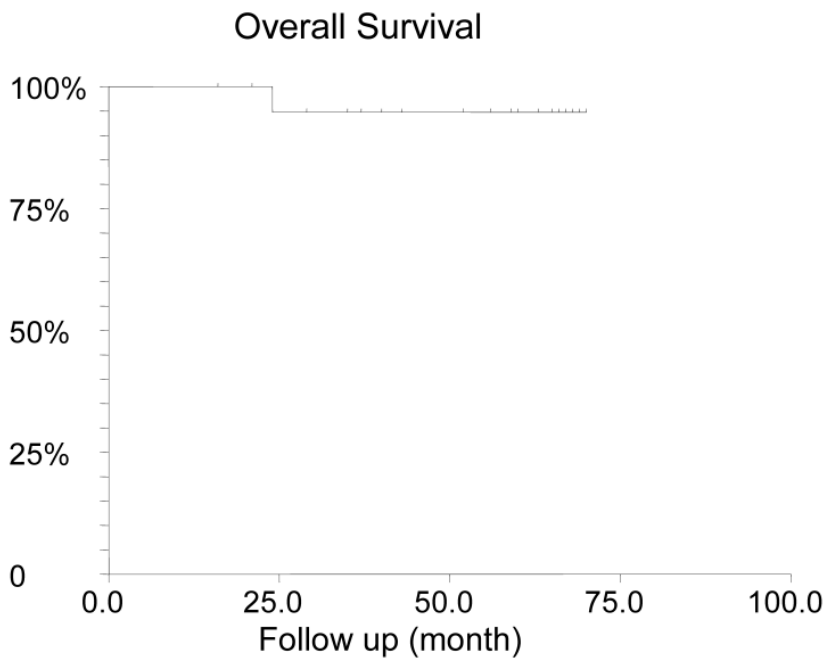

Figure 2. Overall survival
Safety and Tolerability: No patients had progressive disease after two cycles of chemotherapy. All patients proceeded to receive a full course of treatment and were assessable for toxicity. Patients tolerated the chemotherapy well. Nineteen patients (95\%) completed all six cycles of TEC chemotherapy. One patient had five cycles of chemotherapy. The early discontinuation was due to emotional stress but not due to adverse effects. One hundred twenty five $(99.2 \%)$ of 126 planned cycles of TEC were administered. No dose reduction of epirubicin or cyclophosphamide was required. For three patients $(15 \%)$, the dose of docetaxel was reduced by $25 \%$ after cycle 3,4 , and 5 respectively because of grade 3 peripheral neuropathy. Any adverse event, irrespective of whether it is directly caused by chemotherapy, was taken into account (Table 2). Two patients had grade 1-2 tachycardia and 1 patient had palpitation. No patient developed cardiac failure. The most common grade 3-4 adverse effects were neutropenia, 35\%. Three patients (15\%) had neutropenic fever. No death occurred during the treatment.

\section{Discussion}

This phase II study demonstrates that TEC regimen is an efficacious neoadjuvant chemotherapy regimen with a $25 \%$ pCR rate for patients with locally advanced breast cancer. A German study using TEC neoadjuvant chemotherapy regimen recently reported the same pCR rate. ${ }^{5}$ This supports that our results are likely to be reproducible.

The $25 \%$ pCR rate in our study is higher than that of most of the old chemotherapy regimens and is comparable to that of $26 \%$ in NSABP-B27 study ${ }^{6}$ and that of $21 \%$ in GeparTrio trial ${ }^{4}$ (Table 3). In NSABP-B27 study, chemotherapy of AC $x 4$ cycles was followed by docetaxel x 4 cycles. ${ }^{6}$ In GeparTiro trial, patients were given six cycles of TAC chemotherapy. A $21 \%$ pCR rate was obtained from a selected population in which patients demonstrated $\geq 50 \%$ of shrinkage of tumor after two cycles of TAC chemotherapy. ${ }^{4}$ For those who had $<50 \%$ of response after two cycles of TAC treatment and continued to receive six cycles of TAC chemotherapy, the $\mathrm{pCR}$ rate was only $5.3 \% .^{1}$ The definition of pCR was different among these three studies (Table 3). In NSABP-B27 study, pCR was defined as no invasive tumor only in the primary breast site but residual tumor in regional lymph node was allowed. ${ }^{6}$ In GeparTrio trial, pCR was defined as no microscopic evidence of viable tumor (invasive and non invasive) in all resected specimens including breast and regional lymph nodes. ${ }^{4}$ In our study, pCR was defined as absence of invasive tumor in both breast and lymph nodes but in 
situ cancer can be present. If only NSABP-B27 criteria is used for a direct comparison, the $\mathrm{PCR}$ rate of NSABP-B27, GeparTrio and our study would be $26 \%$, $30.6 \%$ and $30 \%$ respectively. In our study, one patient who achieved pCR was not included in the analysis because she had a history of thyroid cancer. Taking account of this patient, the pCR rate would be even higher in our study.

Table 2. Selected Adverse Events on II 9 Cycles of Chemotherapy (\%, any grade $3 / 4$ or $>3$ events for grade 1/2)

\begin{tabular}{|c|c|c|c|}
\hline Events & Grade 1/2 & Grade 3 & Grade 4 \\
\hline nausea/vomiting & 70 & & \\
\hline constipation & 55 & & \\
\hline diarrhea & 40 & 10 & \\
\hline mucositis & 35 & & \\
\hline anorexia & 30 & & \\
\hline abdominal pain & 30 & & \\
\hline dyspepsia & 30 & & \\
\hline fatigue & 85 & & \\
\hline mylgia & 25 & 5 & \\
\hline Bone pain & 30 & & \\
\hline Chest pain & 5 & 5 & \\
\hline Hot flash & 45 & 5 & \\
\hline $\begin{array}{l}\text { Port insertion site reac- } \\
\text { tion }\end{array}$ & 15 & 5 & \\
\hline alopecia & 60 & & \\
\hline Cutaneous reaction & 40 & & \\
\hline syncope & & 15 & \\
\hline dizziness & 20 & & \\
\hline headache & 20 & 5 & \\
\hline Peripheral neuropathy & 15 & 15 & \\
\hline Eye irritation & 35 & & \\
\hline Taste change & 20 & & \\
\hline Runny nose & 35 & & \\
\hline insomnia & 35 & & \\
\hline Mood change & 15 & 10 & \\
\hline Yeast infection & 30 & & \\
\hline Sinusitis/URI & 35 & & \\
\hline UTI & 20 & & \\
\hline gastroenteritis & & 10 & \\
\hline dehydration & & 10 & \\
\hline hyperglycemia & 10 & 5 & \\
\hline edema & 20 & & \\
\hline neutropenia & 10 & 10 & 25 \\
\hline lymphopenia & 5 & 10 & 5 \\
\hline Febrile neutropenia & & 15 & \\
\hline anemia & 70 & & \\
\hline
\end{tabular}

The patient characteristics were different among NSABP-B27, GeparTrio and our study. There were more patients with advanced disease in our study. When there was no nodal involvement, only patients with primary tumor $\geq 3 \mathrm{~cm}$ in diameter were eligible. More than $80 \%$ of patients had biopsy proved nodal disease. The NSABP-27 trial included $14 \%$ patients with tumors $\leq 2 \mathrm{~cm}$ and $70 \%$ of node negative disease. ${ }^{6}$ The GeparTrio trial included patients with T1 disease and $47 \%$ of the patients had no nodal involvement $(\mathrm{N} 0)^{4}$. This study had a slightly lower percentage of hormone receptor positive patients and less HER2 $^{+}$patients (Table 3). In NSABP-27 and GeparTrio trial, no patients received trastuzumab therapy. At the time when this trial was designed and implemented, neoadjuvant trastuzumab therapy was not standard of care. All HER2 ${ }^{+}$patients then received one-year adjuvant trastuzumab treatment. The anti-HER2 therapy was given postoperatively, it thus might improve RFS and OS but should not affect pCR rate. The lower proportion of $\mathrm{HER}_{2}{ }^{+}$patients in this study should not contribute to a higher $\mathrm{pCR}$ rate. Conversely, patients with HER2+ tumors are often accompanied with topoisomerase-II amplification and are more sensitive to docetaxel-based ${ }^{7}$ and anthracycline-based therapy.8,9 Two studies have found that the overexpression of HER2 is associated with a higher $\mathrm{pCR}$ rate. ${ }^{10,11}$ Taken together, even our study had more patients with advanced disease, a comparable pCR rate was obtained. This supports that TEC regimen is a very effective neoadjuvant chemotherapy regimen.

The total planned dose of chemotherapy in this study is slightly higher than of NSABP-B27 and GeparTrio (Table 4). Nevertheless, a high completion rate was achieved. Only one patient did not complete the full course of treatment. In NSABP-B27 trial, 5.5\% of patients did not start docetaxel and in total, $21.2 \%$ of patients did not complete the full four course of AC and four course of docetaxel. In our study, $99.2 \%$ of chemotherapy was actually administered compared to that of $92.2 \%$ in GeparTrio trial. It is worthwhile to note that there were more elderly patients in our study. The percentage of patients who are 60 years of age was $30 \%$ in our study, compared to $9.5 \%$ in GeparTrio $^{4}$ and $13.9 \%$ in NSABP-B27. ${ }^{6}$ The main grade 3-4 toxicity of TEC regimen was neutropenia. Its incidence was slightly lower than that in GeparTrio trial, $35 \%$ vs. $42.1 \%^{4}$ but higher than that of $8.6 \%$ in NSABP-B27 study. ${ }^{4,6}$ The incidence of febrile neutropenia in our study was, however higher than GeparTrio trial, $15 \%$ vs. $7.4 \% .{ }^{4}$ In NSABP-B27 study, $7.3 \%$ of patients had febrile neutropenia while receiving AC but $21.2 \%$ patients developed febrile neutropenia 
while receiving docetaxel. ${ }^{6}$ (Table 4 ) There were more grade 3 peripheral neuropathy in our study than GeparTrio and NSABP-B27 study, $15 \%$ vs. $1.3 \%^{4}$ vs. $2.3 \% .4,6$ Therefore the TEC regimen should be used cautiously for patients with preexisting peripheral neuropathy or patients with high risk to develop neuropathy, such as those with long-term diabetes.

In summary, our single-institution phase II study demonstrated that TEC regimen is an effica- cious and well-tolerated neoadjuvant regimen for patients with locally advanced breast cancer. Future research interest in neoadjuvant chemotherapy in locally advanced breast cancer should be the focus of using genetic profiling such as Oncotype Dx in selecting patients to receive neoadjuvant chemotherapy treatment.

Table 3. Comparison of patient characteristics, design and pCR rate among NSABP-B27, GeparTrio and our study TEC

\begin{tabular}{lllllll}
\hline Trial & Node positive & $\begin{array}{l}\text { Hormone re- } \\
\text { ceptor negative }\end{array}$ & HER2 receptor positive & Planed chemotherapy & $\begin{array}{l}\text { Reported } \\
\text { pCR }\end{array}$ & $\begin{array}{l}\text { Corrected } \\
\text { pCR }\end{array}$ \\
\hline NSABP-B27 & $30.3 \%$ & $38.4 \%^{* *}$ & unknown & $38.1 \%$ & All patients were given 8 cycles & $26 \%$ \\
Gepar Trio & $53 \%$ & $40.1 \%$ & $\begin{array}{l}\text { Only those with tumor size } \\
>50 \% \text { reduction had 6 cycles }\end{array}$ & $21 \%$ & $26 \%$ \\
TEC & $80 \%$ & $50 \%$ & $20 \%$ & All patients were given 6 cycles & $25 \%$ & $30.6 \%$ \\
\hline
\end{tabular}

pCR: pathological complete response

* NSABP-B27 pCR criteria: no invasive disease in the primary tumor was used in all three studies for a comparison.

** $61.8 \%$ of patients have unknown hormone receptor status. For the patients with hormone receptor examined, $38.4 \%$ have negative hormone receptors.

Table 4. Comparison of total planned chemotherapy dosage, completion rate, neutropenia among NSABP-B27, GeparTrio and our study TEC

\begin{tabular}{|c|c|c|c|c|c|c|}
\hline & \multicolumn{3}{|c|}{ Total chemotherapy dosage (mg) } & \multirow[t]{2}{*}{ Completion rate } & \multirow{2}{*}{$\begin{array}{l}\text { Grade 3-4 neutro- } \\
\text { penia }\end{array}$} & \multirow{2}{*}{$\begin{array}{l}\text { Febrile neutro- } \\
\text { penia }\end{array}$} \\
\hline Trial & Docetaxel & Anthracycline & Cyclophosphamide & & & \\
\hline NSABP-B27 & 400 & Doxorubicin 240 & 2,400 & unknown & $8.6 \%$ & $\begin{array}{l}\text { AC } 7.3 \% \\
\text { T } 21.2 \%\end{array}$ \\
\hline Gepar Trio & 450 & Doxorubicin 300 & 3,000 & $92.2 \%$ & $42.1 \%$ & $7.4 \%$ \\
\hline TEC & 450 & Epirubicin 450 & 3,600 & $99.2 \%$ & $35 \%$ & $15 \%$ \\
\hline
\end{tabular}

\section{Competing Interests}

The authors have declared that no competing interest exists.

\section{References}

1. von Minckwitz G, Kummel S, Vogel P, et al. Neoadjuvant vinorelbine-capecitabine versus docetaxel-doxorubicin-cyclophosphamide in early nonresponsive breast cancer: phase III randomized GeparTrio trial. J Natl Cancer Inst. 2008;100:542-551.

2. Rastogi P, Anderson SJ, Bear HD, et al. Preoperative chemotherapy: updates of National Surgical Adjuvant Breast and Bowel Project Protocols B-18 and B-27. I Clin Oncol. 2008;26:778-785.

3. Green MC, Buzdar AU, Smith T, et al. Weekly paclitaxel improves pathologic complete remission in operable breast cancer when compared with paclitaxel once every 3 weeks. J Clin Oncol. 2005;23:5983-5992.
4. von Minckwitz G, Kummel S, Vogel P, et al. Intensified neoadjuvant chemotherapy in early-responding breast cancer: phase III randomized GeparTrio study. I Natl Cancer Inst. 2008;100:552-562.

5. Vogt UKD, Brandt B, Bosse U, Bonk U, Assen AVD, Muhs HJ, Schlotter CM. Semi-quantitative gene expression profiling for therapy prediction in a breast cancer neoadjuvant therpay applying docetaxel/epirubicin/cyclophosphamide. ASCO Annual Meeting Proceedings 2007;25(18S):21144.

6. Bear HD, Anderson S, Brown A, et al. The effect on tumor response of adding sequential preoperative docetaxel to preoperative doxorubicin and cyclophosphamide: preliminary results from National Surgical Adjuvant Breast and Bowel Project Protocol B-27. J Clin Oncol. 2003;21:4165-4174.

7. Martin M, Pienkowski T, Mackey J, et al. Adjuvant docetaxel for node-positive breast cancer. N Engl J Med. 2005;352:2302-2313.

8. Buzdar AU. Topoisomerase IIalpha gene amplification and response to anthracycline-containing adjuvant chemotherapy in breast cancer. J Clin Oncol. 2006;24:2409-2411. 
9. Di Leo A, Isola J. Topoisomerase II alpha as a marker predicting the efficacy of anthracyclines in breast cancer: are we at the end of the beginning? Clin Breast Cancer. 2003;4:179-186.

10. Toi M, Nakamura S, Kuroi K, et al. Phase II study of preoperative sequential FEC and docetaxel predicts of pathological response and disease free survival. Breast Cancer Res Treat. 2008;110:531-539.

11. Rody A, Karn T, Gatje R, et al. Gene expression profiling of breast cancer patients treated with docetaxel, doxorubicin, and cyclophosphamide within the GEPARTRIO trial: HER-2, but not topoisomerase II alpha and microtubule-associated protein tau, is highly predictive of tumor response. Breast. 2007;16:86-93. 